\title{
Ligand-Dependent Activation of Slo Family Channels Is Defined by Interchangeable Cytosolic Domains
}

\author{
Xiao-Ming Xia, Xue Zhang, and Christopher J. Lingle \\ Department of Anesthesiology, Washington University School of Medicine, St. Louis, Missouri 63110
}

\begin{abstract}
Large-conductance $\mathrm{Ca}^{2+}$ - and voltage-regulated $\mathrm{K}^{+}$channels (Slo1 BK-type) are controlled by two physiological stimuli, membrane voltage and cytosolic $\mathrm{Ca}^{2+}$. Regulation by voltage is similar to that in voltage-dependent $\mathrm{K}^{+}$channels, arising from positively charged amino acids primarily within the $\mathrm{S} 4$ transmembrane helices. The basis for regulation by $\mathrm{Ca}^{2+}$ remains controversial. One viewpoint suggests that the extensive cytosolic $\mathrm{C}$ terminus contains the $\mathrm{Ca}^{2+}$ regulatory machinery, whereas another suggests that the pore-forming module contains the $\mathrm{Ca}^{2+}$-sensing elements. To address this issue, we take advantage of another Slo family member, the pH-regulated homolog Slo3. We reason that if the ligand-sensing apparatus is uniquely associated with a particular domain (either the pore or the cytosolic domain), exchange of those domains between Slo1 and Slo3 should result in exchange of ligand dependence in association with the key domain. The results show that the Slo3 cytosolic module confers $\mathrm{pH}$-dependent regulation on the Slo1 pore module, whereas the Slo1 cytosolic module confers $\mathrm{Ca}^{2+}$-dependent regulation on the Slo3 pore module. Thus, ligand-specific regulation is defined by interchangeable cytosolic regulatory modules.
\end{abstract}

Key words: BK channels; calcium [Ca]; potassium; channel; oocyte; patch-clamp; $\mathrm{Ca}^{2+}$ dependence; Slo1; Slo3

\section{Introduction}

Of the ion channels in the voltage-dependent $\mathrm{K}^{+}$channel family, large-conductance $\mathrm{Ca}^{2+}$ - and voltage-regulated $\mathrm{K}^{+}$channels (Slo1 BK-type) are largely unique in the extent to which channel opening is independently regulated by two physiological signals: membrane voltage and cytosolic $\mathrm{Ca}^{2+}$ (Barrett et al., 1982; Moczydlowski and Latorre, 1983). Understanding how two distinct physiological signals each regulate channel opening is likely to be particularly informative about the mechanical linkages that can act either independently or in concert to influence channel activation. The general mechanism of voltage regulation of Slo1 channels is shared with voltage-dependent $\mathrm{K}^{+}$channels (Cui et al., 1997; Horrigan et al., 1999; Cui and Aldrich, 2000) and arises primarily from charged residues within the $\$ 4$ transmembrane segment (Cui et al., 1997; Diaz et al., 1998; Horrigan et al., 1999; Cui and Aldrich, 2000) contained within the pore domain of each $\alpha$ subunit. In contrast, there has been conflicting evidence concerning the sites and mechanisms that may account for physiological regulation by $\mathrm{Ca}^{2+}$. Many studies suggest that C-terminal regulatory structures are critical (Schreiber and Salkoff, 1997; Schreiber et al., 1999; Bian et al., 2001; Moss and Magleby, 2001; Shi et al., 2002; Xia et al., 2002; Magleby, 2003), whereas one study suggests that the pore-forming part of the channel is sufficient to confer $\mathrm{Ca}^{2+}$-dependent activation (Piskorowski and Aldrich, 2002).

Received April 6, 2004; revised May 7, 2004; accepted May 10, 2004

This work was supported by National Institutes of Health Grant GM066215. We declare that we have no competing financial interests. We thank members of C.J.L.'s laboratory for encouragement and assistance during this work.

Correspondence should be addressed to Dr. Christopher J. Lingle, Department of Anesthesiology, Washington University School of Medicine, Box 8054, St. Louis, M0 63110. E-mail: clingle@morpheus.wustl.edu.

DOI:10.1523/JNEUROSCI.1296-04.2004

Copyright $\odot 2004$ Society for Neuroscience $\quad$ 0270-6474/04/245585-07\$15.00/0
After the pore domain, the Slo1 $\alpha$ subunit contains an extensive $\mathrm{C}$ terminus that includes two regulator of conductance for potassium (RCK) domains (Jiang et al., 2002) (see Fig. 1A). RCK domains contain a conserved pattern of $\alpha$ helices and $\beta$ sheets found in a variety of prokaryotic and eukaryotic channel proteins (Jiang et al., 2001, 2002). In many cases, such domains define binding sites for regulatory ligands. Recently, a crystal structure of a $\mathrm{Ca}^{2+}$-regulated bacterial (methanobacterium thermoautotrophicum) $\mathrm{K}^{+}$channel (MthK) revealed a cytosolic module composed of an octamer of RCK domains (Jiang et al., 2002). Each of the four MthK $\alpha$ subunits that contribute to the poreforming module of the channel is associated with a dimer of RCK domains. Remarkably, a pair of RCK domains occurs in each Slo 1 $\alpha$ subunit (Jiang et al., 2002), suggesting not only that the structure of the Slo cytosolic domain may be similar to the MthK structure (see Fig. $1 B$ ) but also that each may share common mechanisms of channel regulation. In Slo1 channels, mutational analysis has shown that the $\mathrm{C}$ terminus contains residues within both the first (Shi et al., 2002; Xia et al., 2002) and second (Schreiber and Salkoff, 1997; Bao et al., 2004) RCK domains that influence channel regulation by $\left[\mathrm{Ca}^{2+}\right]$. However, other work has suggested that the Slo1 pore-forming module may be sufficient to allow $\mathrm{Ca}^{2+}$-dependent channel activation (Piskorowski and Aldrich, 2002). Therefore, it is critical to determine whether the cytosolic domain of the channel plays a fundamental role in ligand dependence.

To address this issue, we take advantage of a $\mathrm{pH}$-regulated but $\mathrm{Ca}^{2+}$-insensitive homolog, Slo3 (Schreiber et al., 1998). If ligand dependence arises specifically from the cytosolic structure, exchange of cytosolic structures between homologous proteins might allow exchange of ligand dependence. In contrast, if ligand dependence arises from the transmembrane components of the 
channel, ligand specificity might associate with the pore modules. Here we successfully exchange the cytosolic regulatory modules between Slo1 and Slo3 pore domains and show that the Slo3 cytosolic module confers robust regulation by cytosolic $\mathrm{pH}$ on the Slo1 pore module. Similarly, the Slo1 cytosolic module confers high-affinity $\mathrm{Ca}^{2+}$ regulation on the Slo3 pore module. The simplest explanation for the results is that ligand-specific gating within the Slo family is defined by the cytosolic regulatory modules.

\section{Materials and Methods}

Generation and expression of chimeric and mutant subunits. The parent constructs for these studies were mouse Slo1 (mSlo1) (Butler et al., 1993) and mSlo3 (Schreiber et al., 1998), both generously provided by L. Salkoff (Washington University, St. Louis, MO). Numbering used here for mSlo1 residues begins with the second potential initiation site. Chimeras 1P3C (Slo1 pore-Slo3 cytosolic domain; mSlo1, 1-347; mSlo3, 337-1121) and 3P1C (Slo3 pore-Slo1 cytosolic domain; mSlo3, 1-315; mSlo1, 327-1169) were generated by standard overlapping PCR methods as used previously in our laboratory (Zeng et al., 2003).

The 5D5N mutation in chimera 3P1C involved residues corresponding to residues 898-902 in mSlo1 (Schreiber and Salkoff, 1997). In 3P1C, this mutation corresponds to residues 886-890. The D362 and D367 residues in Slo1 correspond to residues D351 and D356 in 3P1C. Construction of point mutations was accomplished using standard procedures (Xia et al., 1999, 2002). Channels were expressed in Xenopus oocytes after cRNA injection as described previously (Zhang et al., 2001; Xia et al., 2002).

Percentage identities between Slo1 and Slo3 are expressed as percentage of residues in Slol that are identical to residues in the corresponding positions in the Slo3 sequence.

Physiological recordings. Channel currents were measured from insideout patches (Hamill et al., 1981), as performed routinely in our laboratory (Lingle et al., 2001; Zhang et al., 2001). The pipette extracellular solution contained the following (in $\mathrm{mM}$ ): 140 potassium methanesulfonate, $20 \mathrm{KOH}, 10 \mathrm{HEPES}\left(\mathrm{H}^{+}\right)$, and $2 \mathrm{MgCl}_{2}$, titrated with methanesulfonic acid to a $\mathrm{pH}$ of 7.0. Solutions bathing the cytoplasmic face of the patch membrane contained the following (in $\mathrm{mm}$ ): 140 potassium methanesulfonate, $20 \mathrm{KOH}, 10 \mathrm{mM} \operatorname{HEPES}\left(\mathrm{H}^{+}\right)$, and either 5 EGTA (for nominally $0 \mathrm{Ca}^{2+}$ ) or no added $\mathrm{Ca}^{2+}$ buffer (for $\geq 30 \mu \mathrm{M} \mathrm{Ca}{ }^{2+}$ ). For solutions in which $\mathrm{pH}$ was manipulated, no $\mathrm{Ca}^{2+}$ buffer was used, so that changes in $\mathrm{pH}$ would not alter the effective free $\left[\mathrm{Ca}^{2+}\right]$. $\mathrm{pH}$ was adjusted with either methanesulfonic acid or $\mathrm{KOH}$, and 10 mM HEPES was retained as the buffer in all solutions. Excised patches were bathed in continuously flowing streams from a multibarrel local application system.

For Slo1 and 1P3C, conductance-voltage $(G-V)$ curves were generated from tail currents (Zhang et al., 2001). Slo3 and 3P1C currents exhibit a marked rectification such that tail currents were too small for reliable use in the generation of $G-V$ curves. Therefore, for these constructs $G-V$ curves were generated from steady-state currents, assuming a $0 \mathrm{mV}$ reversal potential (symmetrical $\mathrm{K}^{+}$solutions). At positive potentials, currents for channels containing a Slo3 pore module (either Slo3 or 3P1C) also exhibit strong $\mathrm{Ca}^{2+}$ block such that activation in the absence of channel block cannot be directly measured. Both Slo3 and 3P1C were more than an order of magnitude more sensitive to block of outward current by $\mathrm{Ca}^{2+}$ at $+200 \mathrm{mV}$ than either Slo1 or 1P3C, indicating a unique blocking effect of $\mathrm{Ca}^{2+}$ on the Slo3 pore. However, the shape of the $G-V$ curves at more negative activation potentials allowed estimates of shifts in the activation curves. In all cases, each $G-V$ curve represents average conductance estimates from a set of patches for a given condition. For any patch, conductances at any given ionic condition were normalized to the maximal conductance observed over all conditions. When appropriate, $G-V$ curves were fit with the following:

$$
G(V)=G_{\max } /\left(1+\exp ^{z F\left(V-V_{h}\right) / R T}\right),
$$

to provide estimates of $V_{\mathrm{h}}$, which is the voltage of half activation, and $z$, which is the slope factor describing the voltage dependence of the closed-
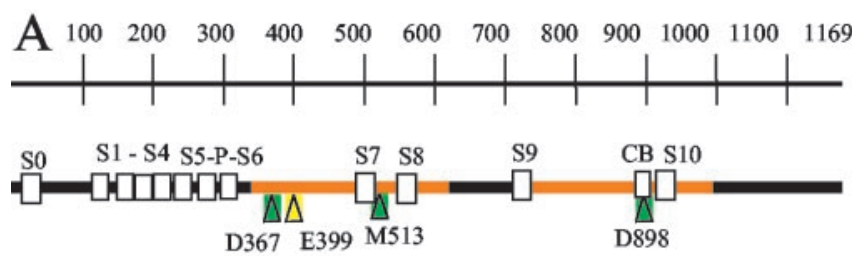

\section{[ RCK1 domain ] [ RCK2 domain ]}
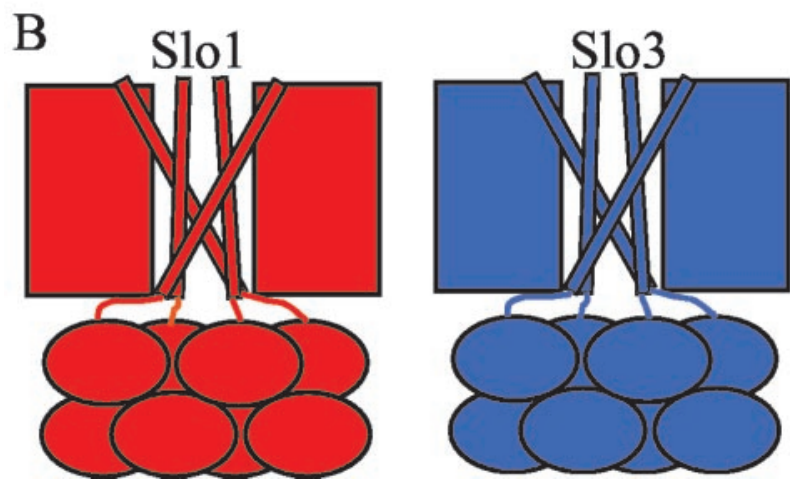

Figure 1. Slo1 and Slo3 channels exhibit a modular structure. A, A linear map of the Slo1 $\alpha$ subunit identifies the transmembrane segments (S1-S6) and pore loop (P) shared with voltage-dependent $\mathrm{K}^{+}$channels in addition to its unique $\mathrm{N}$-terminal $\mathrm{SO}$ segment. An extensive cytosolic C-terminal elaboration contains hydrophobic segments S7-S10 (red segments), the $\mathrm{Ca}^{2+}$ bowl (CB), and two RCK domains (RCK1 and RCK2). Residues implicated in $\mathrm{Ca}^{2+}$ dependent (D367, M513, D898) and $\mathrm{Mg}^{2+}$-dependent (E399) activation are indicated. $B$, Based on homologies with the MthK channel (Jiang et al., 2002), an octameric cytosolic domain arising from two RCK domains in each $\alpha$ subunit is appended to the membrane-embedded pore module.

open equilibrium. $V_{\mathrm{h}}$ estimates for a given ionic condition represent the mean value for a set of 5-10 individual patches. In all cases, error bars indicate SEM. Experiments were at room temperature $\left(21-24^{\circ} \mathrm{C}\right)$. Most salts and chemicals were from Sigma (St. Louis, MO). We were unable to obtain currents or single-channel openings ( $n=16$ patches) from a construct corresponding to the Slo1 pore module truncated at the same position as in previous work (Piskorowski and Aldrich, 2002). In this set of patches, recordings were typically maintained in excess of $15 \mathrm{~min}$, with no evidence of channel activity.

\section{Results}

Slo1 currents are regulated by $\mathrm{Ca}^{2+}$, but not by $\mathrm{pH}$, and $\mathrm{Slo} 3$ currents are regulated by $\mathrm{pH}$, but not by $\mathrm{Ca}^{2+}$

A linear representation of the Slo1 sequence is provided in Figure $1 A$ to highlight key elements important for the present work. Each Slo1 $\alpha$ subunit consists of two discrete structural domains (Fig. 1 $A, B$ ). The pore-forming domain consists of transmembrane segments S0-S6, which share extensive homology to voltage-gated $\mathrm{K}^{+}$channels. After the S6 inner helix, an extensive cytosolic $\mathrm{C}$ terminus contains a pair of segments with homology to RCK domains (Jiang et al., 2001, 2002). For the Slo1 channel, residues in two distinct locations of the $\mathrm{C}$ terminus influence regulation by micromolar $\mathrm{Ca}^{2+}$ (for review, see Magleby, 2003). One location is the so-called $\mathrm{Ca}^{2+}$ bowl (Schreiber and Salkoff, 1997; Bao et al., 2004), a sequence of aspartate residues (D897D891) found within the second RCK domain of the C terminus (Fig. $1 A$ ). In addition, residues in the first RCK domain [D367 (Xia et al., 2002) and M513 (Bao et al., 2002)] (Fig. 1 A) remove a second component of regulation by micromolar $\mathrm{Ca}^{2+}$, whereas residue $\mathrm{E} 399$ has been implicated in regulation by millimolar $\mathrm{Mg}^{2+}$ (Shi et al., 2002; Xia et al., 2002). Together, D367 and mutation of all aspartate residues in the $\mathrm{Ca}^{2+}$ bowl (termed 


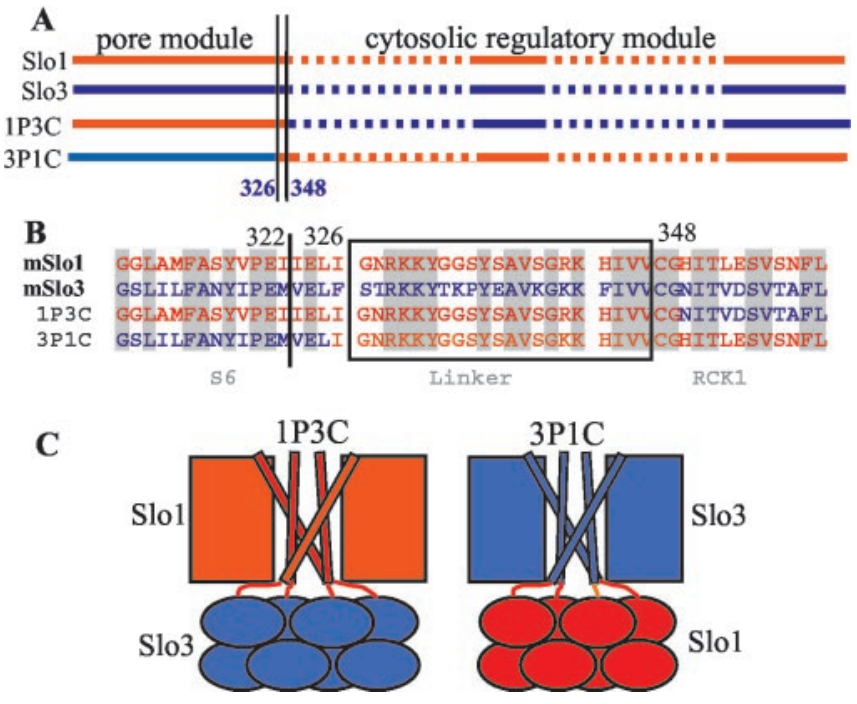

Figure 2. Representations of constructs used to study ligand dependence of Slo channel activation. A, Linear maps of the Slo1, Slo3, 1P3C, and 3P1C constructs used here. Residue numbers correspond to Slo1. Cytosolic domains from Slo1 and Slo3 were swapped to create constructs $1 \mathrm{P} 3 \mathrm{C}$ and $3 \mathrm{P} 1 \mathrm{C}$. Note that both chimeric constructs contain identical linkers between $\$ 6$ and the cytosolic domain. $B$, The sequences of each construct through the boundaries between segments. The last residue (I322) in the truncated Slo 1 is also noted (Piskorowski and Aldrich, 2002). C, The general organization of pore and cytosolic regulatory modules for the $1 \mathrm{P} 3 \mathrm{C}$ and $3 \mathrm{P} 1 \mathrm{C}$ chimera.

$5 \mathrm{D} 5 \mathrm{~N})$ remove all regulation by $\mathrm{Ca}^{2+}$ concentrations of $<1 \mathrm{mM}$ (Xia et al., 2002). Yet, a truncated Slo1 construct with a stop codon at residue position 323 (Fig. 2B) just after the S6 inner helix has been reported to form rarely occurring channels that exhibit regulation by $\mathrm{Ca}^{2+}$ (Piskorowski and Aldrich, 2002), although we have been unable to record such channels (see Materials and Methods). As an alternative approach to defining the essential elements necessary for ligand dependence in Slo family channels, here we have examined the consequences of exchanging cytosolic domains between Slo1 and Slo3. We begin by first defining basic aspects of the $\mathrm{Ca}^{2+}$ and $\mathrm{pH}$ dependence of both Slo1 and Slo3.

Slo1 currents exhibit a characteristic shift in activation with elevations in cytosolic $\left[\mathrm{Ca}^{2+}\right]$ (Cox et al., 1997; Cox and Aldrich, 2000; Zhang et al., 2001). Typically, $V_{\mathrm{h}}$ obtained from $G-V$ curves shifts to more negative potentials as $\mathrm{Ca}^{2+}$ is elevated (Fig. $3 A$ ). In contrast, changes in $\mathrm{pH}$ from 7.0 to 8.0 have minor effects on Slo1 $G-V$ curves either at $0 \mathrm{Ca}^{2+}$ or at $300 \mu \mathrm{M} \mathrm{Ca}{ }^{2+}$ (Fig. 3B), with only a small enhancement at lower $\mathrm{pH}$ (Avdonin et al., 2003). For Slo3, increases in cytosolic $\mathrm{pH}$ from 7.0 to 8.0 result in marked activation of current (Schreiber et al., 1998) (Fig. 3C,E), with only minimal current activation at a $\mathrm{pH}$ of 7.0. In contrast, increases in $\left[\mathrm{Ca}^{2+}\right]$ at a $\mathrm{pH}$ of 7.0 do not increase current activation. To examine the effects of $\mathrm{Ca}^{2+}$ more closely, the effect of $0-300 \mu \mathrm{M}$ $\left[\mathrm{Ca}^{2+}\right]$ was examined at a $\mathrm{pH}$ of 8.0. No shift in activation of conductance was observed, although extensive blockade of Slo3 channels by $\mathrm{Ca}^{2+}$ was observed at more positive potentials (Fig. $3 D)$. At voltages for which block by $\mathrm{Ca}^{2+}$ was minimal $(0$ to +50 $\mathrm{mV})$, current activation by 0 and $300 \mu \mathrm{M} \mathrm{Ca}^{2+}$ was indistinguishable. Thus, Slo3 channels are robustly regulated by $\mathrm{pH}$ but not by $\mathrm{Ca}^{2+}$.

\section{The Slo3 cytosolic domain confers $\mathrm{pH}$ dependence on a Slo1} pore module

We created chimeric constructs in which the C termini of Slo1 and Slo3 were joined, respectively, with the pore-defining mod-

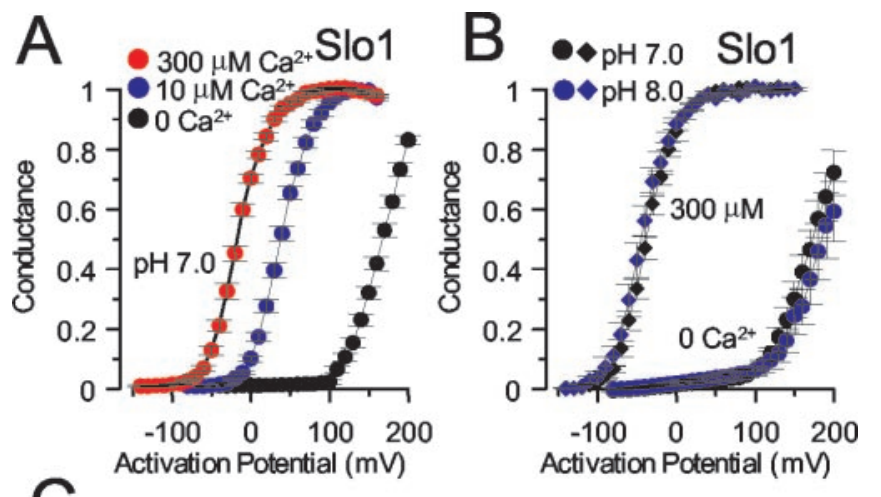

C
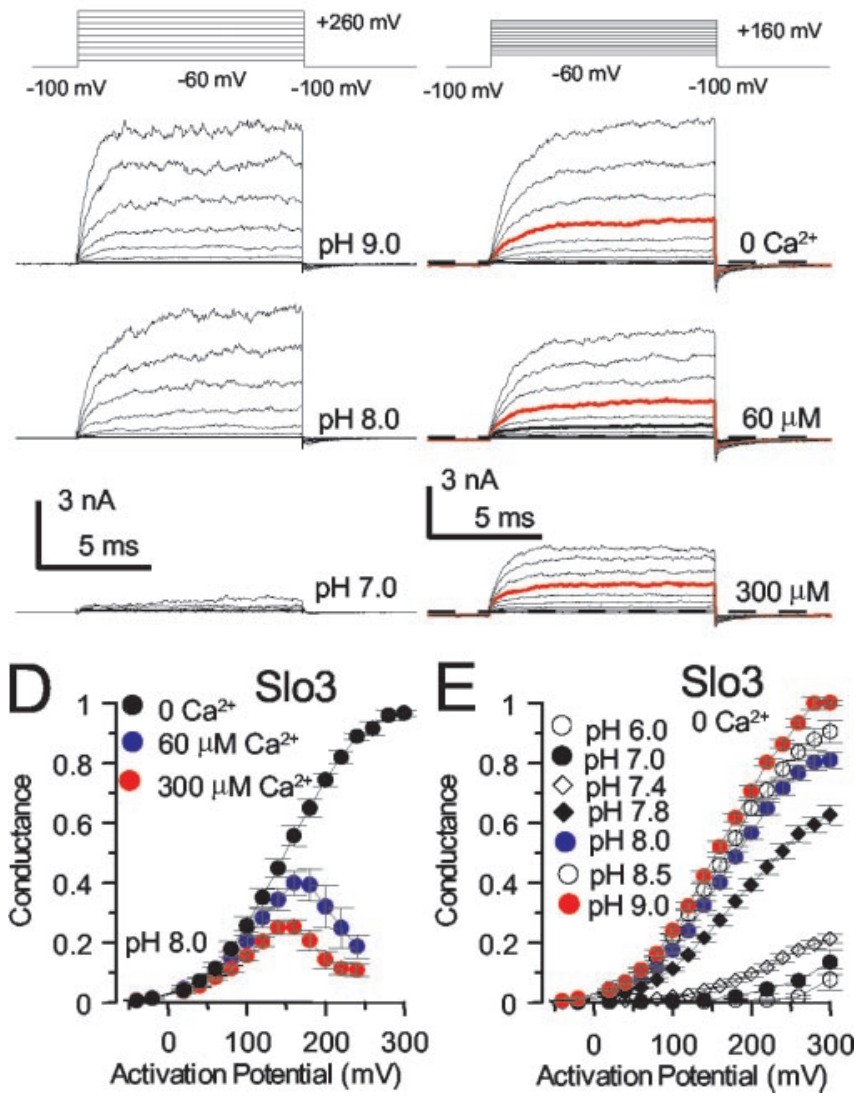

Figure 3. Slo1 and Slo3 are regulated by distinct cytosolic factors. $A$, Increases in $\left[\mathrm{Ca}^{2+}\right]$ shift the activation range of $\mathrm{Sl} 01$ channels. Each curve is a $\mathrm{G}-V$ relationship at a specific $\left[\mathrm{Ca}^{2+}\right]$. $B$, Increases in pH from 7.0 to 8.0 have minor effects on activation of Slo 1 current at either 0 or $300 \mu \mathrm{Ma}^{2+}$. C, Increases in pH cause a marked enhancement of Slo3 current activation, as shown for $\mathrm{pH}$ values of 7.0, 8.0, and 9.0 (left, with $0 \mu \mathrm{M} \mathrm{Ca}^{2+}$ ), whereas increases in $\mathrm{Ca}^{2+}$ from 0 to $300 \mu \mathrm{m}$ have no activating effect (right, with a pH of 8.0). Red traces correspond to currents activated at $+100 \mathrm{mV}$ to emphasize the lack of effect of $\mathrm{Ca}^{2+}$ on conductance. D, G-V curves obtained from peak Slo3 outward currents at different $\left[\mathrm{Ca}^{2+}\right]$ values at a $\mathrm{pH}$ of 8.0 illustrate the lack of effect of $\mathrm{Ca}^{2+}$ on $\mathrm{Sl} 03$ currents. E, G-V curves obtained from peak Slo3 currents at different $\mathrm{pH}$ values with $0 \mathrm{Ca}^{2+}$ illustrate the marked activation of conductance produced by increases in $\mathrm{pH}$.

ules of Slo3 and Slo1 (Fig. 2A-C). In construct 1P3C, the Slo3 C terminus was appended to a Slo1 pore-forming domain. In construct 3P1C, the Slo1 cytosolic domain was appended to the Slo3 pore. In both cases, the linker between the pore module and the cytosolic module was from Slo1 (Fig. 2B).

Patches from oocytes expressing 1P3C channels showed minimal current activation with depolarizations to $+200 \mathrm{mV}$ at a $\mathrm{pH}$ of 7.0 for $\left[\mathrm{Ca}^{2+}\right]$ up through $10 \mathrm{~mm}$. In contrast, as the $\mathrm{pH}$ was 


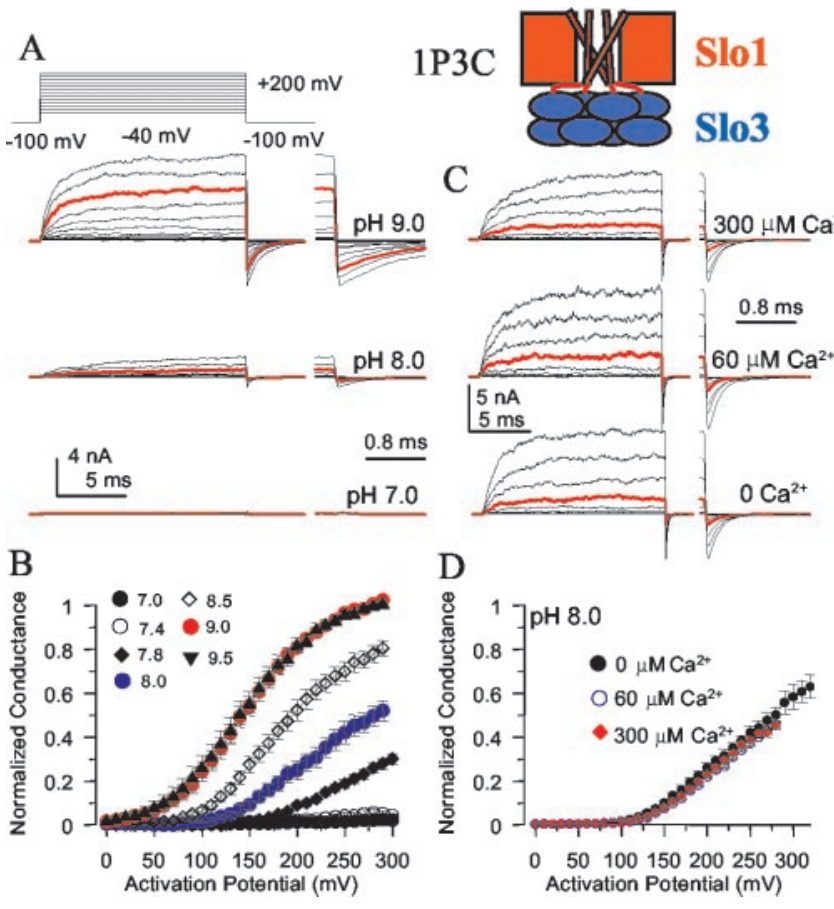

Figure 4. The Slo3 cytosolic module confers pH dependence on the Slo1 pore module. $A$, Currents resulting from $1 \mathrm{P} 3 \mathrm{C}$ are regulated by $\mathrm{pH}$. On the right, tail currents are shown at a faster base. Red traces highlight current activated at $+80 \mathrm{mV}, 0 \mathrm{Ca}^{2+} . \mathrm{B}, \mathrm{G}-\mathrm{V}$ curves resulting from $1 \mathrm{P3C}$ current activation as a function of $\mathrm{pH}$ with $0 \mathrm{Ca}^{2+}$. Conductances were normalized within each patch to the maximum conductance observed at elevated pH. C, 1P3C currents (activated by the same voltage protocol as in $A$ and with a pH of 8.0) are not activated by $\mathrm{Ca}^{2+}$. The red trace highlights current activated at a $\mathrm{pH}$ of 8.0. D, G-V curves resulting from $1 \mathrm{P} 3 \mathrm{C}$ current activation as a function of $\mathrm{Ca}^{2+}, \mathrm{pH}$ 8.0. Elevated $\mathrm{Ca}^{2+}$ produces a small inhibition of outward current but no shift in activation.

increased above 7.4 (with $0 \mathrm{Ca}^{2+}$ ), activation of current became appreciable (Fig. $4 A$ ), with a shift to more negative potentials at higher $\mathrm{pH}$ values (Fig. $4 \mathrm{~B}$ ). Thus, $\mathrm{pH}$ dependence generally similar to that of wild-type Slo3 was conferred on the Slo1 pore module by the Slo3 cytosolic domain, arguing that $\mathrm{pH}$ dependence of both 1P3C and Slo3 channels arises from that domain.

The absence of an effect of $\mathrm{Ca}^{2+}$ on $1 \mathrm{P} 3 \mathrm{C}$ at a $\mathrm{pH}$ of 7.0 might result from the very positive voltages required for current activation. 1P3C currents were therefore studied with 0,60 , or $300 \mu \mathrm{M}$ $\left[\mathrm{Ca}^{2+}\right]$ at a $\mathrm{pH}$ of 8.0. Except for some blocking effects of $300 \mu \mathrm{M}$ $\mathrm{Ca}^{2+}$ on peak outward current (Fig. 4C), G-V curves were essentially identical at each $\left[\mathrm{Ca}^{2+}\right]$ concentration. This is markedly in contrast to the approximately $-180 \mathrm{mV}$ shift (Xia et al., 2002) in $V_{\mathrm{h}}$ resulting from the same change in $\left[\mathrm{Ca}^{2+}\right]$ for Slo1 (Fig. $3 A$ ). Thus, the Slo3 cytosolic domain confers $\mathrm{pH}$ sensitivity but not $\mathrm{Ca}^{2+}$ sensitivity on the Slol pore module. The inability of $\mathrm{Ca}^{2+}$ to promote activation of $1 \mathrm{P} 3 \mathrm{C}$ suggests either that the critical $\mathrm{Ca}^{2+}$ binding site is absent or that a $\mathrm{Ca}^{2+}$ binding site on the pore domain is unable to regulate the channel. Yet $1 \mathrm{P} 3 \mathrm{C}$ is strongly regulated by $\mathrm{pH}$, indicating that the $\mathrm{Slo} 3 \mathrm{C}$ terminus does permit robust regulation of gating of the Slo1 pore.

These results argue against the possibility that the $\mathrm{pH}$ dependence of Slo3 arises simply from proton inhibition of current flow by an action on the Slo3 permeation pathway. Although protons reduce the single-channel conductance of Slo1 channels at positive potentials (Brelidze and Magleby, 2004), Slo1 tailcurrent amplitudes are minimally affected by $\mathrm{pH}$ values of $\geq 7.0$ at negative potentials. Thus, the fact that the $1 \mathrm{P} 3 \mathrm{C}$ channels contain the relatively $\mathrm{pH}$-insensitive $\mathrm{Slo} 1$ pore module, while retain-
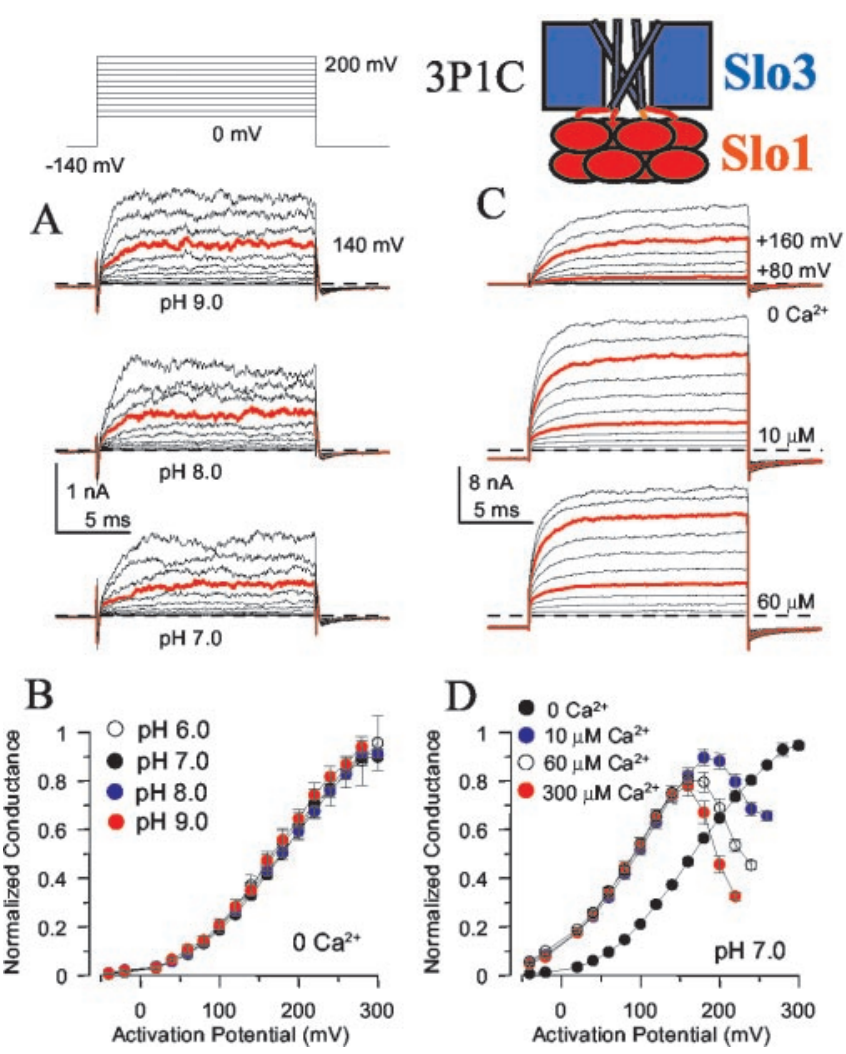

Figure 5. The Slo1 C terminus confers sensitivity to micromolar $\mathrm{Ca}^{2+}$ on the Slo3 pore module. $A, 3 \mathrm{P} 1 C$ currents were activated by the indicated voltage protocol over a range of $\mathrm{pH}$ values from 7.0 to 9.0. $\mathrm{Ca}^{2+}$ was buffered to low levels with $5 \mathrm{~mm}$ EGTA. Red traces highlight currents at $+140 \mathrm{mV}$ to emphasize the lack of effect of increases in $\mathrm{pH}$ on current activation. $B$, $G-V$ curves were generated from outward currents through 3P1C channels obtained at various $\mathrm{pH}$ values (all with $0 \mathrm{Ca}^{2+}$ ). C, Activation of $3 \mathrm{P} 1 \mathrm{C}$ currents is shifted by increases in $\mathrm{Ca}^{2+}$. Red traces highlight currents activated at +80 and $+160 \mathrm{mV}$ to emphasize the increase in conductance at a given voltage. $D, G-V$ curves show activation of $3 \mathrm{P} 1 \mathrm{C}$ currents at different $\mathrm{Ca}^{2+}$ values (all at a pH of 7.0). For $0 \mathrm{Ca}^{2+}, V_{\mathrm{h}}=165.7 \pm 3.1 \mathrm{mV}, \mathrm{z}=0.50 \pm 0.01, n=20$; for 10 $\mu \mathrm{MCa}{ }^{2+}, V_{\mathrm{h}}=97.4 \pm 3.5 \mathrm{mV}, \mathrm{z}=0.57 \pm 0.31, n=19$.

ing regulation by $\mathrm{pH}$, argues that it is the $\mathrm{Slo} 3$ cytosolic domain that confers $\mathrm{pH}$ sensitivity on both $\mathrm{Slo} 3$ and 1P3C.

\section{A Slo1 cytosolic module confers $\mathrm{Ca}^{2+}$ dependence on a Slo3 pore module}

We subsequently examined whether the Slo1 cytosolic domain confers $\mathrm{Ca}^{2+}$-dependent regulation on the $\mathrm{Ca}^{2+}$-insensitive Slo3 pore module (construct 3P1C). 3P1C currents were activated by standard voltage protocols at a $\mathrm{pH}$ of 7.0 with $0 \mathrm{Ca}^{2+}$. Changes in pH from 6.0 to 9.0 had no effect on current activation (Fig. 5A,B). In contrast, $10 \mu \mathrm{M} \mathrm{Ca}^{2+}$ resulted in an appreciable increase in 3P1C current activation, with no obvious additional effect of 60 or $300 \mu \mathrm{M} \mathrm{Ca}^{2+}$ (Fig. 5C); a $10 \mu \mathrm{M}$ concentration of $\mathrm{Ca}^{2+}$ produces an $\sim 70 \mathrm{mV}$ negative shift in $G-V$ curves compared with 0 $\mathrm{Ca}^{2+}$ (Fig. 5D). As with Slo3, such estimates are complicated by extensive $\mathrm{Ca}^{2+}$-dependent block of outward current. However, the ability of $\mathrm{Ca}^{2+}$ to shift $G-V$ curves in 3P1C (Fig. 5D) clearly differs from the results with Slo3 (Fig. 3D). Thus, the Slo1 cytosolic module confers regulation by micromolar $\mathrm{Ca}^{2+}$ on the Slo3 pore module, whereas $\mathrm{pH}$-dependent regulation is absent. These observations argue strongly that regulation by micromolar $\mathrm{Ca}^{2+}$ arises from the Slol cytosolic module, whereas the $\mathrm{pH}$ dependence arises from the Slo3 cytosolic module. 

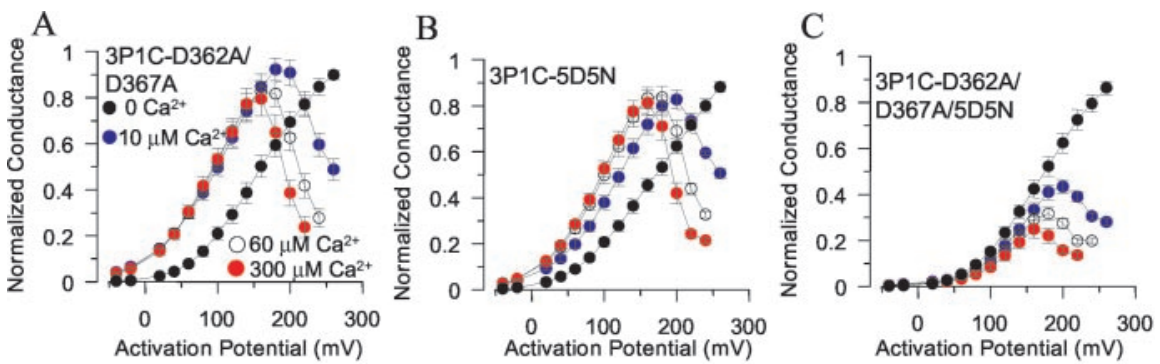

Figure 6. $\mathrm{Ca}^{2+}$ dependence of $3 \mathrm{P} 1 \mathrm{C}$ activation is abolished by mutations that abolish $\mathrm{Ca}^{2+}$ dependence of Slo1 activation. $A$, G-V curves resulting from activation of 3P1C-D362A,D367A channels are shown for $\left[\mathrm{Ca}^{2+}\right]$ from 0 to $300 \mu \mathrm{m}$. At $0 \mathrm{Ca}^{2+}(n=7)$, $V_{\mathrm{h}}=161.3 \pm 8.3 \mathrm{mV}, \mathrm{z}=0.58 \pm 0.02$; at $10 \mu \mathrm{m} \mathrm{Ca}{ }^{2+}(n=7), V_{\mathrm{h}}=102.3 \pm 4.5 \mathrm{mV}, z=0.66 \pm 0.05 . B, \mathrm{G}-V$ curves resulting from activation of $3 \mathrm{P} 1 \mathrm{C}-5 \mathrm{D} 5 \mathrm{~N}$ are shown for $\left[\mathrm{Ca}^{2+}\right]$ from 0 to $300 \mu \mathrm{m}$. At $0 \mathrm{Ca}^{2+}(n=7), V_{\mathrm{h}}=173.8 \pm 7.7 \mathrm{mV}, z=0.48 \pm$ 0.02 ; at $10 \mu \mathrm{M} \mathrm{Ca}^{2+}(n=7), V_{\mathrm{h}}=122.0 \pm 6.8 \mathrm{mV}, z=0.59 \pm 0.04$. C, G-V curves resulting from activation of 3P1C$\mathrm{D} 362 \mathrm{~A}, \mathrm{D} 367 \mathrm{~A}, 5 \mathrm{D} 5 \mathrm{~N}$ show that $\mathrm{Ca}^{2+}$-dependent activation has been abolished. At $0 \mathrm{Ca}^{2+}(n=12), V_{\mathrm{h}}=178.1 \pm 8.4 \mathrm{mV}, \mathrm{z}=$ $0.58 \pm 0.02$; at $10 \mu \mathrm{M} \mathrm{Ca}^{2+}(n=12), V_{\mathrm{h}}=193.0 \pm 7.0 \mathrm{mV}, z=0.57 \pm 0.02$.
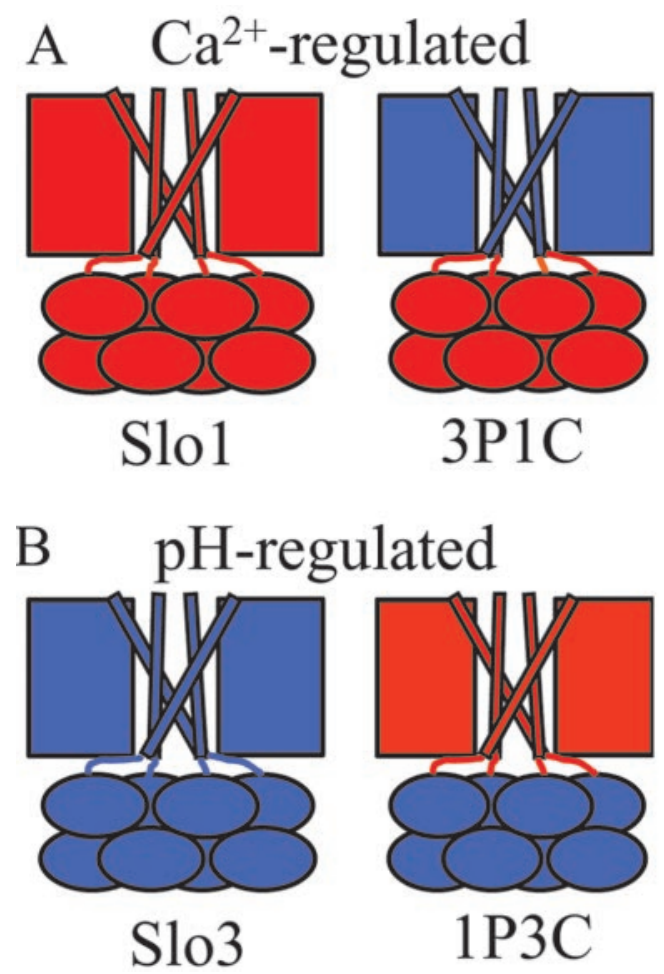

Figure 7. $\mathrm{Ca}^{2+}$-dependent activation arises from the Slo1 cytosolic domain, whereas $\mathrm{pH}-$ dependent regulation is associated with the Slo3 cytosolic domain. $A$, Constructs that exhibit regulation by $\mathrm{Ca}^{2+}$, but not by pH, share a Slo1 cytosolic domain. $B$, Constructs that exhibit regulation by $\mathrm{pH}$, but not by $\mathrm{Ca}^{2+}$, share a Slo1 cytosolic domain.

\section{Residues in Slo1 that influence $\mathrm{Ca}^{2+}$ dependence play similar roles in 3P1C channels}

The magnitude of the shift in gating produced by $\mathrm{Ca}^{2+}$ on 3P1C differs from that seen for wild-type Slo1. Specifically, in Slo1 an increase in $\left[\mathrm{Ca}^{2+}\right]$ from 0 to $10 \mu \mathrm{M}$ produces an $\sim 130 \mathrm{mV}$ shift to more negative activation potentials, with an additional negative shift of $\sim 55 \mathrm{mV}$ through $300 \mu \mathrm{M}$ (Xia et al., 2002). In contrast, for 3P1C we observe an $\sim 70 \mathrm{mV}$ negative shift in $V_{\mathrm{h}}$ for a $0-10 \mu \mathrm{M}$ increase in $\left[\mathrm{Ca}^{2+}\right]$, with an additional shift of only a few millivolts with increases to $300 \mu \mathrm{M}$. The smaller shift caused by $\mathrm{Ca}^{2+}$ in 3P1C may not be surprising, because in a complex allosteric protein (Rothberg and Magleby, 1999; Cox and Aldrich, 2000; Cui and Aldrich, 2000; Zhang et al., 2001; Horrigan and Aldrich,
2002), $\mathrm{Ca}^{2+}$ binding may simply be less effective at coupling to channel activation in the chimeric construct. The magnitude of the shift in $V_{\mathrm{h}}$ produced by a given increment in $\left[\mathrm{Ca}^{2+}\right]$ cannot be compared simply among widely differing constructs (Bao et al., 2002). However, the difference does raise questions as to whether wildtype Slo1 $\mathrm{Ca}^{2+}$ dependence has been fully restored and whether the $\mathrm{Ca}^{2+}$ dependence of 3P1C arises from mechanisms similar to those in Slo1. Therefore, we asked whether mutations that influence the $\mathrm{Ca}^{2+}$ sensitivity of Slo1 also influence the $\mathrm{Ca}^{2+}$ sensitivity of 3P1C. For Slo1, mutation of aspartate residues (5D5N) in the so-called $\mathrm{Ca}^{2+}$ bowl region removes one portion of the sensitivity to micromolar $\mathrm{Ca}^{2+}$ (Schreiber and Salkoff, 1997; Xia et al., 2002). In addition, mutation of residues (D362A,D367A) in the first Slo1 RCK domain removes a second portion of the sensitivity to micromolar $\mathrm{Ca}^{2+}$, whereas the combined D362A,D367A,5D5N construct exhibits no sensitivity to $\mathrm{Ca}^{2+}$ up to $1 \mathrm{~mm}$ (Xia et al., 2002).

Figure 6 shows the consequences of these mutations in the 3P1C construct. For the D362A,D367A mutation (in 3P1C), 10 $\mu \mathrm{M} \mathrm{Ca}^{2+}$ shifts activation similar to its effects on 3P1C (Fig. 6A). For the 5D5N mutation (in 3P1C), the ability of $10 \mu \mathrm{M} \mathrm{Ca}^{2+}$ to shift activation appears somewhat reduced compared with 3P1C, but in contrast to $3 \mathrm{P} 1 \mathrm{C}$, increases in $\mathrm{Ca}^{2+}$ to 60 and $300 \mu \mathrm{M} \mathrm{Ca}^{2+}$ result in additional leftward $G-V$ shifts (Fig. $6 B$ ). For the simultaneous D362A,D367A,5D5N mutation, the ability of $\mathrm{Ca}^{2+}$ to shift activation was abolished (Fig. $6 \mathrm{C}$ ), leaving only the $\mathrm{Ca}^{2+}$ dependent blockade characteristic of constructs containing the Slo3 pore domain. The fact that both mutations together are required to abolish fully the sensitivity of 3P1C to micromolar $\mathrm{Ca}^{2+}$ is identical to the effects of these mutations on Slo1 (Xia et al., 2002) and argues that both RCK1 residues and the $\mathrm{Ca}^{2+}$ bowl in RCK2 contribute to regulation by $\mathrm{Ca}^{2+}$ in $3 \mathrm{P} 1 \mathrm{C}$.

\section{Discussion}

The results demonstrate that for both Slo1 and Slo3, regulation by specific cytosolic factors arises from the C-terminal structure that follows the S6 inner helix. Specifically, the C-terminal cytosolic domain from $\mathrm{pH}$-sensitive Slo3, when appended to the Slo1 pore domain, confers regulation by $\mathrm{pH}$ on the resulting chimeric channel (1P3C). Similarly, the C-terminal cytosolic domain from $\mathrm{Ca}^{2+}$-sensitive Slo1, when appended to the Slo3 pore domain, confers regulation by $\mathrm{Ca}^{2+}$ on the resulting chimeric channel (3P1C). The simplest explanation for these observations is that ligand dependence and ligand binding in the Slo family of proteins arise from the extensive C-terminal regulatory domains (Fig. 7). This explanation also seems most consistent with the idea that the RCK domains of bacterial $\mathrm{K}^{+}$channels define regulatory structures for a variety of cytosolic ligands (Jiang et al., 2001, 2002), including nucleotides and ions.

Our results do not provide an explanation for the observation that a truncated form of the BK channel, essentially identical to the Slo1 pore module, may appear in the plasma membrane and result in functional $\mathrm{Ca}^{2+}$-dependent channels (Piskorowski and Aldrich, 2002). Could ligand regulation of the $1 \mathrm{P} 3 \mathrm{C}$ and $3 \mathrm{P} 1 \mathrm{C}$ chimeras studied here actually be defined by the respective poreforming modules of Slo1 and Slo3, but the appended C termini simply permit regulation by ligand to occur? Such a possibility 
would require an extremely complex model. If the function of the $\mathrm{C}$ terminus is unrelated to ligand recognition, one would expect that ligand dependence for any construct should be defined by the pore-forming domain of the channel. Alternatively, one might imagine that ligand dependence arising from the pore module might be suppressed because of an inappropriate C-terminal regulatory structure. Contrary to these possibilities, for sensitivity either to micromolar $\mathrm{Ca}^{2+}$ or to $\mathrm{pH}$, we observed that ligand-dependent regulation was based solely on identity of the $\mathrm{C}$ terminus (Fig. 7). Thus, the extensive $\mathrm{C}$-terminal regulatory domain is the core element that appears to define the physiologically significant ligand dependence in the Slo family of proteins.

Although both chimeric constructs exhibit unique ligand dependence characteristic of the identity of their cytosolic structure, details of the ligand dependence do not exactly mirror that of wild-type Slo1 and Slo3. Specifically, the shifts in activation produced by micromolar $\mathrm{Ca}^{2+}$ in 3P1C are approximately half of those observed in Slo1, although the range of $\mathrm{Ca}^{2+}$ concentrations that produce shifts is similar in both cases. Similarly, with regard to the $\mathrm{pH}$ dependence of $\mathrm{Slo} 3$ and $1 \mathrm{P} 3 \mathrm{C}$, although both exhibit robust increases in channel activation with elevations in $\mathrm{pH}$, there appear to be differences in the $\mathrm{pH}$ dependence of those increases. For Slo3, some activation of current can be observed at $+300 \mathrm{mV}$ with a $\mathrm{pH}$ as low as 6.0 , whereas for the chimeric 1P3C, little current activation is observed until at least $\mathrm{pH}$ 7.4. Despite these differences in the ability of $\mathrm{Ca}^{2+}$ or $\mathrm{pH}$ to regulate gating in wild-type versus chimeric constructs, however, the difference in effectiveness is probably not surprising in such large, complex allosteric proteins. The energetics of opening of the pore domain are likely to be quite different between Slo1 and Slo3, such that a given cytosolic regulatory domain may be differentially effective depending on the nature of the pore domain.

Another difference that was observed between Slo1 and 3P1C was the consequences of mutation of D362A,D367A and 5D5N. In Slo1, the D362A,D367A and 5D5N mutations behave in an approximately energetically additive manner (Xia et al., 2002), whereas in 3P1C, the results of Figure 6 suggest that there may be some interactions between the two sites. Regardless of this interesting difference, these results are consistent with the view that the regulation by micromolar $\mathrm{Ca}^{2+}$ conferred on the Slo3 pore module by the Slo1 cytosolic domain has a structural and functional basis similar to that of the wild-type Slo1 channel. Regulation of Slo1 and 3P1C channels by micromolar $\mathrm{Ca}^{2+}$ appears to arise from similar determinants on the common Slo1 C-terminal cytosolic domain. Additional work will be required to address the possibility that the two regions of the cytosolic structure that have been implicated in $\mathrm{Ca}^{2+}$-dependent regulation may interact in some way.

A remarkable aspect of these results is the exchangeable modularity of the ligand regulatory elements (i.e., that $\mathrm{pH}$ and $\mathrm{Ca}^{2+}$ dependence can be exchanged between distinct pore domains). Although Slo1 and Slo3 share $\sim 40 \%$ aa identity, the correspondence is $\sim 63 \%$ in the pore domain and $\sim 37 \%$ in the cytosolic domains. The fact that specific ligand recognition is associated with the cytosolic structure suggests that most of the key elements of that ligand regulatory process, including binding and the conformational changes required to influence channel gating, are intrinsic to that domain. Yet both cytosolic domains, exhibiting only $\sim 37 \%$ identity, are able to regulate activation of a foreign pore domain. One possibility is that all pore and cytosolic domains retain key conserved residues that preserve liganddependent regulation of the pore. However, the fact that rela- tively divergent cytosolic modules can each permit regulation of a foreign pore domain may suggest that the ligand regulatory machinery, whether it involves the $\mathrm{pH}$ sensitivity of the Slo3 cytosolic domain or the $\mathrm{Ca}^{2+}$ sensitivity of the Slo1 cytosolic domain, may exert its effects by a generalized mechanism that may not be strongly dependent on a set of specific interactions between the pore domain and the cytosolic structure. Such a view would be consistent with the model proposed for regulation of MthK gating by the octamer of RCK domains (Jiang et al., 2002). The hypothesis proposed for gating of the MthK channel suggests that rotation of the dimers of RCK domains produces a change in tension on a helical linker connecting the cytosolic structures to the S6 inner helices (Jiang et al., 2002). This tension on the linker is proposed to provide the energy to favor the movement of the S6 inner helix into an open-channel conformation. As long as the pore domain does not place constraints on the ability of tension applied on the linker to change the $\mathrm{S} 6$ conformation, the ability of a cytosolic domain to regulate gating of a foreign pore domain may be preserved.

The idea that the Slo1 $\alpha$ subunit may be a complex of distinct functional modules has also been suggested in previous work (Wei et al., 1994), in which completely normal currents were obtained from the separate expression of two distinct cRNAs, each containing separate portions of the $\mathrm{C}$ terminus. One message corresponded approximately to the pore domain with the first half of the cytosolic domain and the other corresponded to the second half of the cytosolic domain. The justification for the separation into two parts was that a comparison of Drosophila Slo1 and mSlo1 suggested that the $\mathrm{C}$ terminus consisted of two regions of relatively strong conservation with an intervening section of residues exhibiting length and residue mismatch. Now it is clear that the two more conserved regions of the $\mathrm{C}$ terminus correspond, in general, to the two RCK domains, which appear to be connected by a linker of lesser functional importance. It is remarkable that the expression of the Slo1 channel in parts so closely mirrors what may occur naturally for the MthK channel (Jiang et al., 2002). The MthK gene encodes a pore-forming sequence along with a single C-terminal RCK domain. However, a secondary initiation methionine located between the pore sequence and the RCK domain results in the expression of two peptides: one containing a pore sequence and a single RCK domain and the second containing only an RCK domain (Jiang et al., 2002). Functional channels are proposed to arise from the assembly of the four individual RCK domains with a tetramer of pore-forming subunits. Thus, the previous demonstration that Slo1 channels can arise from expression by parts can now be seen as consistent with the proposed octameric arrangement of RCK domains in both MthK and Slo1 channels.

The mammalian family of Slo-related genes consists of four members. In addition to the $\mathrm{Ca}^{2+}$-regulated Slo1 (Adelman et al., 1992; Butler et al., 1993) and the pH-regulated Slo3 (Schreiber et al., 1998), two additional homologs have been identified, Slo2.1 (Bhattacharjee et al., 2003), also termed Slick, and Slo2.2 (Joiner et al., 1998; Yuan et al., 2000, 2003), also termed Slack. Slo2.2 subunits are regulated by both $\mathrm{Na}^{+}$and $\mathrm{Cl}^{-}$(Yuan et al., 2003) and perhaps by $\mathrm{Ca}^{2+}$ (Yuan et al., 2000), and Slo2.1 subunits, although less well characterized, also share sensitivity to $\mathrm{Na}^{+}$and $\mathrm{Cl}^{-}$(Bhattacharjee et al., 2003). Thus, the hallmark of the Slo family of channels appears to be regulation by distinct cytosolic ligands. Based on the results presented here, it appears that the cytosolic domain of each Slo family channel defines that unique ligand dependence. 


\section{References}

Adelman JP, Shen KZ, Kavanaugh MP, Warren RA, Wu YN, Lagrutta A, Bond CT, North RA (1992) Calcium-activated potassium channels expressed from cloned complementary DNAs. Neuron 9:209-216.

Avdonin V, Tang X, Hoshi T (2003) Stimulatory action of internal protons on Slo1 BK channels. Biophys J 84:2969-2980.

Bao L, Rapin A, Holmstrand E, Cox D (2002) Elimination of the $\mathrm{BK}_{\mathrm{Ca}}$ channel's high affinity $\mathrm{Ca}^{2+}$ sensitivity. J Gen Physiol 120:173-189.

Bao L, Kaldany C, Holmstrand E, Cox D (2004) Mapping the BKCa channel's " $\mathrm{Ca}^{2+}$ bowl": side-chains essential for $\mathrm{Ca}^{2+}$ sensing. J Gen Physiol 123:475-489.

Barrett JN, Magleby KL, Pallotta BS (1982) Properties of single calciumactivated potassium channels in cultured rat muscle. J Physiol (Lond) 331:211-230.

Bhattacharjee A, Joiner WJ, Wu M, Yang Y, Sigworth FJ, Kaczmarek LK (2003) Slick (Slo2.1), a rapidly-gating sodium-activated potassium channel inhibited by ATP. J Neurosci 23:11681-11691.

Bian S, Favre I, Moczydlowski E (2001) $\mathrm{Ca}^{2+}$-binding activity of a COOHterminal fragment of the Drosophila BK channel involved in $\mathrm{Ca}^{2+}$ dependent activation. Proc Natl Acad Sci USA 98:4776-4781.

Brelidze TI, Magleby KL (2004) Protons block BK channels by competitive inhibition with $\mathrm{K}^{+}$and contribute to the limits of unitary currents at high voltages. J Gen Physiol 123:305-319.

Butler A, Tsunoda S, McCobb DP, Wei A, Salkoff L (1993) mSlo, a complex mouse gene encoding "maxi” calcium-activated potassium channels. Science 261:221-224.

Cox D, Aldrich R (2000) Role of the $\beta 1$ subunit in large-conductance $\mathrm{Ca}^{2+}$ activated $\mathrm{K}^{+}$channel gating energetics: mechanisms of enhanced $\mathrm{Ca}^{2+}$ sensitivity. J Gen Physiol 116:411-432.

Cox DH, Cui J, Aldrich RW (1997) Allosteric gating of a large conductance Ca-activated $\mathrm{K}^{+}$channel. J Gen Physiol 110:257-281.

Cui J, Aldrich RW (2000) Allosteric linkage between voltage and $\mathrm{Ca}^{2+}$. dependent activation of BK-type mSlol $\mathrm{K}^{+}$channels. Biochemistry 39:15612-15619.

Cui J, Cox DH, Aldrich RW (1997) Intrinsic voltage dependence and $\mathrm{Ca}^{2+}$ regulation of mSlo large conductance Ca-activated $\mathrm{K}^{+}$channels. J Gen Physiol 109:647-673.

Diaz L, Meera P, Amigo J, Stefani E, Alvarez O, Toro L, Latorre R (1998) Role of the $\mathrm{S} 4$ segment in a voltage-dependent calcium-sensitive potassium (hSlo) channel. J Biol Chem 273:32430-32436.

Hamill OP, Marty A, Neher E, Sakmann B, Sigworth FJ (1981) Improved patch-clamp techniques for high-resolution current recording from cells and cell-free membrane patches. Pflügers Arch 391:85-100.

Horrigan F, Aldrich R (2002) Coupling between voltage-sensor activation, $\mathrm{Ca}^{2+}$ binding and channel opening in large conductance (BK) potassium channels. J Gen Physiol 120:267-305.

Horrigan FT, Cui J, Aldrich RW (1999) Allosteric voltage gating of potassium channels. I. mSlo ionic currents in the absence of $\mathrm{Ca}^{2+}$. J Gen Physiol 114:277-304.

Jiang Y, Pico A, Cadene M, Chait BT, MacKinnon R (2001) Structure of the RCK domain from the E. coli $\mathrm{K}^{+}$channel and demonstration of its presence in the human BK channel. Neuron 29:593-601.

Jiang Y, Lee A, Chen J, Cadene M, Chait BT, MacKinnon R (2002) Crystal structure and mechanism of a calcium-gated potassium channel. Nature 417:515-522.

Joiner WJ, Tang MD, Wang LY, Dworetzky SI, Boissard CG, Gan L, Gribkoff VK, Kaczmarek LK (1998) Formation of intermediate-conductance calcium-activated potassium channels by interaction of Slack and Slo subunits. Nat Neurosci 1:462-469.

Lingle CJ, Zeng X-H, Ding J-P, Xia X-M (2001) Inactivation of BK channels mediated by the $\mathrm{N}$-terminus of the $\beta 3 \mathrm{~b}$ auxiliary subunit involves a twostep mechanism: possible separation of binding and blockade. J Gen Physiol 117:583-605.

Magleby KL (2003) Gating mechanism of BK (Slo1) channels: so near, yet so far. J Gen Physiol 121:81-96.

Moczydlowski E, Latorre R (1983) Gating kinetics of $\mathrm{Ca}^{2+}$-activated $\mathrm{K}^{+}$ channels from rat muscle incorporated into planar lipid bilayers: evidence for two voltage-dependent $\mathrm{Ca}^{2+}$ binding reactions. J Gen Physiol 82:511-542.

Moss BL, Magleby KL (2001) Gating and conductance properties of BK channels are modulated by the S9-S10 tail domain of the alpha subunit: a study of mSlo1 and mSlo3 wild-type and chimeric channels. J Gen Physiol 118:711-734.

Piskorowski R, Aldrich RW (2002) Calcium activation of BK(Ca) potassium channels lacking the calcium bowl and RCK domains. Nature 420:499-502.

Rothberg BS, Magleby KL (1999) Gating kinetics of single largeconductance $\mathrm{Ca}^{2+}$-activated $\mathrm{K}^{+}$channels in high $\mathrm{Ca}^{2+}$ suggest a twotiered allosteric gating mechanism. J Gen Physiol 114:93-124.

Schreiber M, Salkoff L (1997) A novel calcium-sensing domain in the BK channel. Biophys J 73:1355-1363.

Schreiber M, Wei A, Yuan A, Gaut J, Saito M, Salkoff L (1998) Slo3, a novel $\mathrm{pH}$-sensitive $\mathrm{K}^{+}$channel from mammalian spermatocytes. J Biol Chem 273:3509-3516.

Schreiber M, Yuan A, Salkoff L (1999) Transplantable sites confer calcium sensitivity to BK channels. Nat Neurosci 2:416-421.

Shi J, Krishnamoorthy G, Yang Y, Hu L, Chaturvedi N, Harilal D, Qin J, Cui J (2002) Mechanism of magnesium activation of calcium-activated potassium channels. Nature 418:876-880.

Wei A, Solaro C, Lingle C, Salkoff L (1994) Calcium sensitivity of BK-type KCa channels determined by a separable domain. Neuron 13:671-681.

Xia XM, Ding JP, Lingle CJ (1999) Molecular basis for the inactivation of $\mathrm{Ca}^{2+}$ - and voltage-dependent BK channels in adrenal chromaffin cells and rat insulinoma tumor cells. J Neurosci 19:5255-5264.

Xia X-M, Zeng X-H, Lingle CJ (2002) Multiple regulatory sites in largeconductance calcium-activated potassium channels. Nature 418:880-884.

Yuan A, Dourado M, Butler A, Walton N, Wei A, Salkoff L (2000) SLO-2, a $\mathrm{K}^{+}$channel with an unusual $\mathrm{Cl}^{-}$dependence. Nat Neurosci 3:771-779.

Yuan A, Santi CM, Wei A, Wang ZW, Pollak K, Nonet M, Kaczmarek L, Crowder CM, Salkoff L (2003) The sodium-activated potassium channel is encoded by a member of the Slo gene family. Neuron 37:765-773.

Zeng XH, Xia XM, Lingle CJ (2003) Redox-sensitive extracellular gates formed by auxiliary beta subunits of calcium-activated potassium channels. Nat Struct Biol 10:448-454.

Zhang X, Solaro CR, Lingle CJ (2001) Allosteric regulation of BK channel gating by $\mathrm{Ca}^{2+}$ and $\mathrm{Mg}^{2+}$ through a non-selective, low affinity divalent cation site. J Gen Physiol 118:607-635. 